\title{
Metacarpal Neck Fractures: A Review of Surgical Indications and Techniques
}

\author{
Eric M. Padegimas, ${ }^{1,}{ }^{*}$ William J. Warrender, ${ }^{1}$ Christopher M. Jones, ${ }^{2}$ and Asif M. Ilyas ${ }^{2}$ \\ ${ }^{1}$ Department of Orthopedic Surgery, Thomas Jefferson University Hospital, Philadelphia, USA \\ ${ }^{2}$ Department of Orthopedic Surgery, The Rothman Institute, Thomas Jefferson University Hospital, Philadelphia, USA \\ "Corresponding author: Eric M. Padegimas, Department of Orthopedic Surgery, Thomas Jefferson University Hospital, 1025 Walnut Street, Room 516 College, Philadelphia, USA. \\ Tel: +1-2159551500; +1-8606040902, Fax: +1-2155030530, E-mail: padegimase@gmail.com
}

Received 2015 September 04; Accepted 2016 January 26.

\begin{abstract}
Context: Hand injuries are a common emergency department presentation. Metacarpal fractures account for $40 \%$ of all hand fractures and can be seen in the setting of low or high energy trauma. The most common injury pattern is a metacarpal neck fracture. In this study, the authors aim to review the surgical indications for metacarpal neck fractures, the fixation options available along with the risk and benefits of each.

Evidence Acquisition: Literature review of the different treatment modalities for metacarpal neck fractures. Review focuses on surgical indications and the risks and benefits of different operative techniques.

Results: The indications for surgery are based on the amount of dorsal angulation of the distal fragment. The ulnar digits can tolerate greater angulation as the radial digits more easily lose grip strength. The most widely utilized fixation techniques are pinning with k-wires, dorsal plating, or intramedullary fixation. There is currently no consensus on an optimal fixation technique as surgical management has been found to have a complication rate up to $36 \%$. Plate and screw fixation demonstrated especially high complication rates.

Conclusions: Metacarpal neck fractures are a common injury in young and active patients that results in substantial missed time from work. While the surgical indications are well-described, there is no consensus on the optimal treatment modality because of high complication rates. Dorsal plating has higher complication rates than closed reduction and percutaneous pinning, but is necessary in comminuted fractures. The lack of an ideal fixation construct suggests that further study of the commonly utilized techniques as well as novel techniques is necessary.
\end{abstract}

Keywords: Metacarpal Bone, Neck Fractures, Hand, Fracture Fixation, Internal, Bone Wires

\section{Context}

Hand injuries are a common presentation to an emergency department (1). Fractures involving the hand account for up to $28 \%$ of all fractures encountered (2). While the most common fractures of the hand are of the distal phalanx (3), metacarpal fractures alone account for about 40\% of all hand fractures with 1.5 million injuries occurring annually $(4,5)$. They are the second most common fracture to present to an orthopaedic surgeon (after distal radius fractures) with an incidence of 130.3 per 100,000 patients annually (6). The economic burden is particularly high in these patients as they typically affect young and healthy men (7) leading to missed time from work. Isolated metacarpal injuries can result in up to $3-6$ weeks of missed time with non-operative management alone (8-10). Therefore, optimizing treatment of these injuries may have significant benefits in both quality of life and return to work for these patients.
Among metacarpal fractures, the metacarpal neck is the most common site of injury (11). Metacarpal neck fractures typically result from a patient striking a solid surface with a clenched fist causing volar comminution and dorsal apex angulation $(12,13)$. The most frequent scenario encountered is a fracture of fifth metacarpal neck otherwise known as the "boxer's fracture." Metacarpal neck fractures provide a particular dilemma to orthopaedic surgeons as there is no consensus for their management (14, 15). Non-operative management typically delivers an acceptable outcome, but can be associated with a poor cosmetic result, weakness, extensor lag, and palmar prominence (16). Conversely, with surgical intervention there have been reports of high complication rates, up to $36 \%$ (17-19). The controversy surrounding the management of metacarpal neck fractures, prompted review of the treatment options available. 


\section{Evidence Acquisition}

This article will provide an evidence-based review of the presentation and different surgical management options for metacarpal neck fractures. This is intended to clarify the surgical options for these injuries with the specific risks and benefits for each technique. We will review the typical presentation of metacarpal neck fractures, radiographic findings, surgical indications, and fixation techniques available to the surgeon.

PubMed was used as an electronic search engine in this review. The search terms used to identify studies were "metacarpal fractures" and "metacarpal neck fractures". All studies were scrutinized to reflect a wide spectrum of treatment options for metacarpal neck fractures. Additionally, two widely circulated orthopaedic textbooks were utilized for general information on the presentation, radiographic features, surgical indications and fixation techniques.

\section{Results}

\subsection{History and Physical Examination}

A thorough history and physical exam must be done for evaluation of metacarpal neck fractures. Key aspects of the patient history include age, hand dominance and occupation as they can significantly affect management. Mechanism of injury is an important variable for early management and outcome of metacarpal neck fractures. The classic presentation of a metacarpal neck fracture is a patient that strikes a solid surface with a closed fist $(12,13)$. However, these injuries can also occur in multiply injured polytrauma patients. Metacarpal neck fractures can be subtle, as can most hand fractures, in the setting of a multiply injured patient which can lead to delays in diagnosis and treatment (20).

The first component of physical examination is careful inspection of the skin and soft tissue. The clinician must be careful to fully examine for lacerations to rule out an open fracture. Wounds over the metacarpophalangeal (MCP) joints should be considered to communicate with the fracture or the MCP joint until proven otherwise (Figure 1). For less obvious injuries, localized swelling, ecchymosis and tenderness to palpation can be helpful isolating the fracture. MCP joint depression or loss of normal joint contour may be present (Figure 2). If there is a soft tissue defect, the patient should receive both tetanus prophylaxis as well as antibiotic prophylaxis that covers common oral flora (amoxicillin/clavulanic acid), presuming the laceration was from a tooth sustained from punching someone in the mouth, also known as a "fight bite" $(21,22)$.

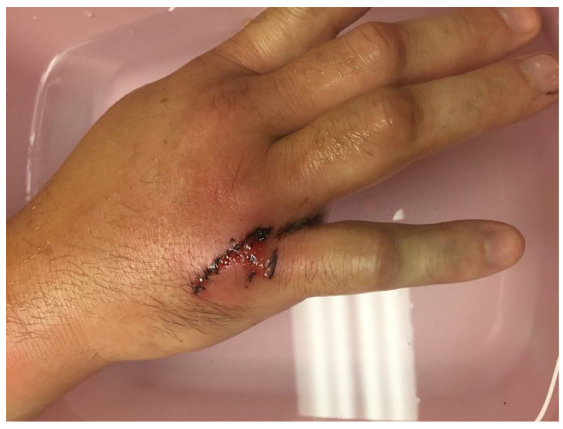

Figure 1. Fight Bite Sustained With Metacarpal Neck Fracture

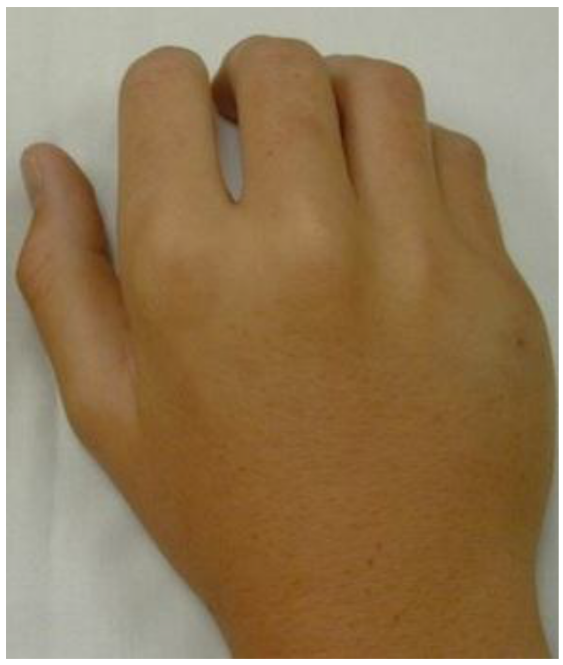

Figure 2. Note the Altered Contour and Deformity of the Fifth Small Finger ray Along the Border due to an Underlying Angulated Metacarpal Neck Fracture

Additionally, the specifics of the deformity can be noted. Shortening of the ray can be assessed by comparison to the contralateral hand. The rotational alignment should also be evaluated. A rotational deformity may not be appreciated with the fingers in extension, however testing them in flexion may reveal subtle deformity. Normally in flexion, the digits will point to the distal pole of the scaphoid, but in metacarpal fractures with rotational deformity, there will be some degree of external rotation of the affected finger (Figure 3). If the patient is unable to actively flex his or her fingers due to pain, this deformity will also be present with passive flexion.

Finally, a neurovascular examination is undertaken prior to any local anesthetic or reduction attempt. Distal capillary refill should be less than two seconds and two point discrimination should be less than $6 \mathrm{~mm}$. Dorsal wounds may be associated with deficits of the dor- 
Figure 3. Note the Rotational Deformity due to an Underlying Spiral Metacarpal Fracture

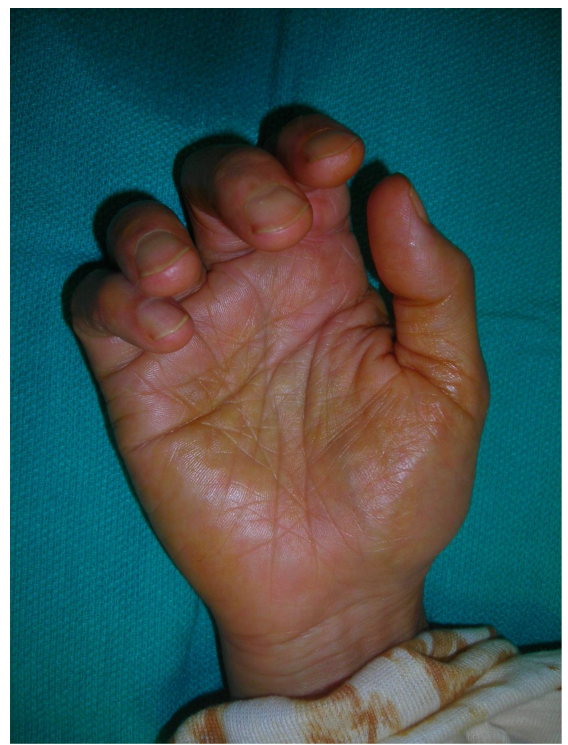

Rotational deformity is most accentuated with active or passive finger flexion, while angular deformity is most prominent with finger extension.

sal sensory branches of the ulnar or radial nerves. Similarly, volar wounds are commonly associated with digital nerve injury. Motor function is checked in the finger flexors and extensors. Extensor tendons can be lacerated and retracted especially when dorsal wounds are present. "Pseudo-clawing" (hyperextension) of the ray may result after repeated attempts to extend the finger if the angulation of the metacarpal neck fracture is advanced $(23,24)$. Evaluation of finger extension and grip strength in the subacute or chronic setting (not acutely) should also be noted.

\subsection{Imaging}

Imaging and subsequent diagnosis of metacarpal neck fractures can be readily achieved with plain radiographs. Anteroposterior, lateral and oblique radiographs of the affected digit should preferably be obtained. A "skyline" view of the metacarpophalangeal joint has been described and can show a vertical impaction defect in the metacarpal head which is typically sustained as a result of tooth penetration through the extensor hood into the joint (25). Injured digits should be viewed individually to minimize overlap of other digits. Additionally, every attempt at a perfect lateral should be made as it has been shown that the obliquity of the hand at the time of radiograph can effect the angulation measurement (26). Advanced imaging is typically not necessary unless there is concern for intra-articular extension of the fracture to the metacarpal head. Articular involvement would best be evaluated by computed tomography (CT).

Imaging should be surveyed for comminution, displacement, angulation, shortening and fractures to surrounding bones of the hand and wrist (Figure 4A - C). There are two different techniques for measuring angulation on the lateral radiograph. In one, the angle is measured between the line along the longitudinal axis of the metacarpal shaft (medullary canal) and the line from the center of the metacarpal head to the fracture site (Figure $4 \mathrm{D})$. In the other, the angle is measured after drawing two lines tangential to the dorsal cortices of the proximal and distal fragments using the intersecting angle between the two lines as the measure of the fracture angulation (this method may underestimate angulation) (Figure 4E). Normal angulation of the 5th metacarpal neck is fifteen degrees using the first of the aforementioned methods (12). The measurement of fracture angulation of small finger metacarpal neck fractures is subject to a high degree of inter- and intraobserver variability (27). Reliability and validity can be adequate only when the degree of small finger metacarpal neck fracture angulation is measured after drawing lines on lateral radiographs. Oblique radiograph measurements consistently produce higher readings (28). Finally, one must account for the fact that the normal metacarpal head-to-neck angle is 15 degrees so any fracture angulation is equal to the measured angle on the lateral radiograph minus 15 degrees.

\subsection{Surgical Indications}

There are a number of surgical indications for metacarpal neck fractures. Firstly, open fractures are an absolute indication for irrigation, debridement, and fracture reduction (29). Secondly, if there is extension of the fracture into the metacarpal head with greater than $1 \mathrm{~mm}$ of displacement or any intra-articular fracture fragment blocking joint motion, surgery is recommended (29). Finally, and most commonly, the degree of volar angulation and displacement is used to determine operative versus nonoperative management. If volar angulation is not corrected, patients can have a prominent dorsal deformity, decreased grip strength, a prominent palmar metacarpal head and pseudo clawing (Figure 2) (23, 24). The degrees of volar angulation for each digit that may be considered for surgical fixation are (29):

-150 for the index finger

- 250 for the long finger

- 350 for the ring finger

- 450 for the small finger

Notably, the angular criteria for surgical intervention is highly variable with some authors accepting up to 700 of angulation $(8,18,30)$. The acceptable degree 
Figure 4. Radiographic Assessment of Metacarpal Neck Fractures, Note the Dorsal Angulation
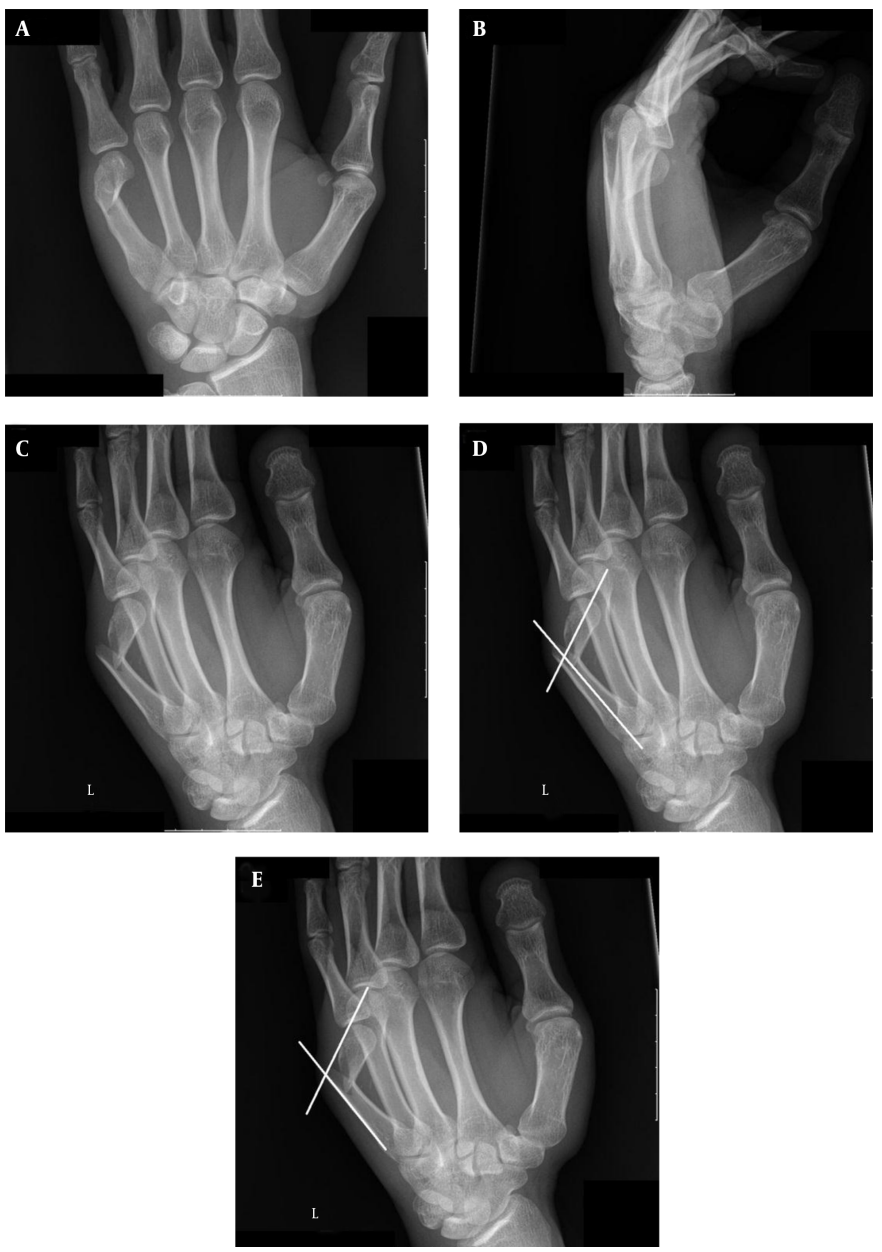

A, B and C, PA, lateral, and oblique X-rays demonstrating displaced, dorsally angulated fifth metacarpal neck fracture; D, measurement of dorsal angulation between the line along the longitudinal axis of the metacarpal shaft (medullary canal) and the line from the center of the metacarpal head to the fracture site; E, measurement of dorsal angulation between the two lines tangential to the dorsal cortices of the proximal and distal fragments using the intersecting angle between the two lines as the measure of the fracture angulation.

of angulation increases for the more ulnar digits owing to the compensatory movement of the 4th and 5th carpometacarpal joints. Less angulation of the second and third metacarpals can be tolerated due to the fact that their CMC joints are much less mobile. When considering operative indications, it is important to note that in the small finger, greater than 30 degrees of angulation is associated with a decrease in flexor digiti minimi grip strength and range of motion (30).

\subsection{Surgical Technique}

\subsubsection{Closed Reduction and Pinning}

Metacarpal neck fractures are reduced by maximal flexion of the metcarpophalangeal (MCP) joint with an axial load directed dorsally across the flexed proximal interphalangeal (PIP) joint. The fracture site can then be pinned by three different techniques.

\subsubsection{Cross Pinning}

This technique utilizes 0.9 or $1.1 \mathrm{~mm}$ K-wires placed distal to the fracture site at the flare of the metacarpal neck or in the collateral recess of the metacarpal head. The pins are advanced in a retrograde fashion bicortically without crossing at the fracture site to control rotation (Figure 5).

\subsubsection{Crucifix Pinning}

This technique utilizes a $1.6 \mathrm{~mm}$ K-wire placed retrograde through the head and into the intermedullary 
Figure 5. Radiographic Assessment of Metacarpal Neck Fracture After Reduction and Cross-pinning
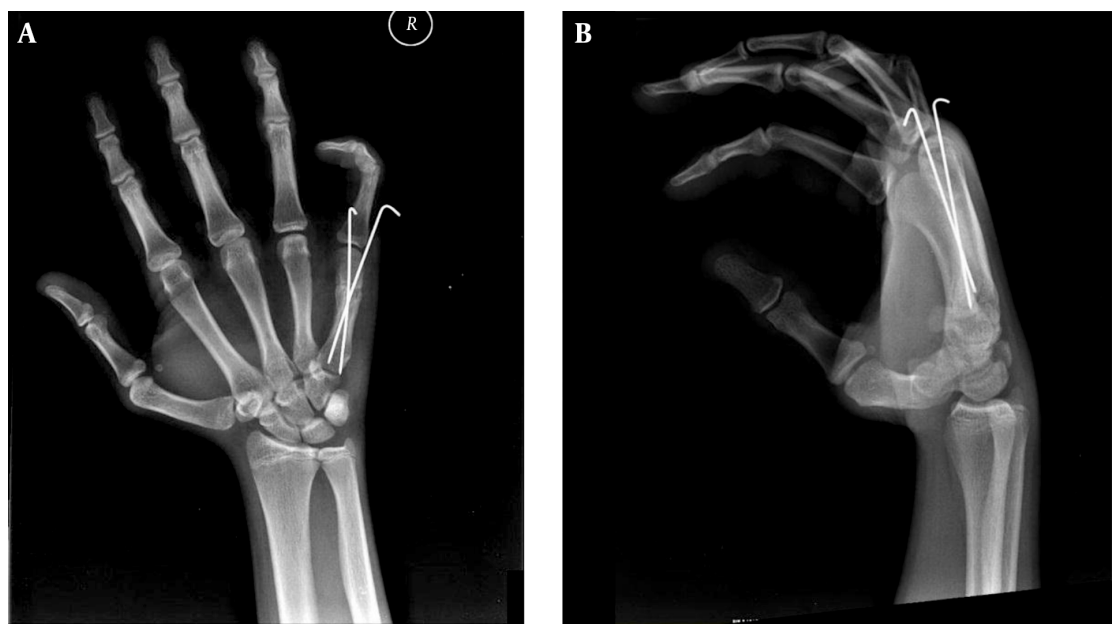

A and B, PA and lateral X-rays demonstrating cross-pinning construct showing reduction of the metacarpal neck fracture.

canal, and then placing a $0.045 \mathrm{~K}$-wire laterally through the metacarpal head and advancing it bicortically into the adjacent metacarpal head to hold the rotation (Figure 6).

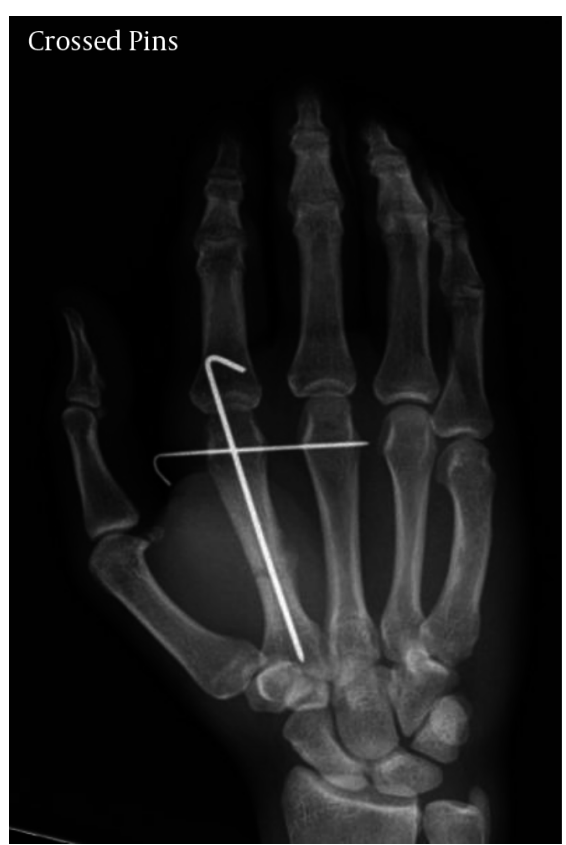

Figure 6. Crucifix Pinning Showing Reduction of the Metacarpal Neck Fracture by Two-crossed Pins

\subsubsection{Bouquet Pinning}

This technique utilizes a "bouquet" of typically three Kwires placed for intramedullary fixation in antegrade fashion. These have a dorsal bend to preferentially support the dorsal aspect of the metacarpal head to hold reduction (Figure 7) (31, 32).

Benefits of the technique include:

- Minimally invasive

- Good post-operative range of motion (97.7\% compared to contralateral side) (33)

Complications/Drawbacks of the technique include:

- Less stable with extensive comminution (29)

- Pinsite infection (34)

- Nonunion/malunion (35)

- Tendon adhesion and joint contracture from prolonged immobilization

- Need to protect exposed pins

\subsubsection{Plate and Screw Fixation}

Plate and screw fixation (open reduction, internal fixation) is performed dorsally with retraction of the extensor tendons and subperiosteal exposure of the metacarpal neck. Partial or complete incision of the sagittal band may be necessary for distal exposure, but should be repaired upon closure. The distal exposure may be limited by the MCP joint capsule, potentially necessitating arthrotomy. With full exposure, the fracture is reduced and a plate is applied with a minimum of two screws placed in the shaft and a minimum of two screws placed into the head and neck. The volar cartilage may prohibit bicortical fixation of the distal fracture fragment. Two potential solutions are 


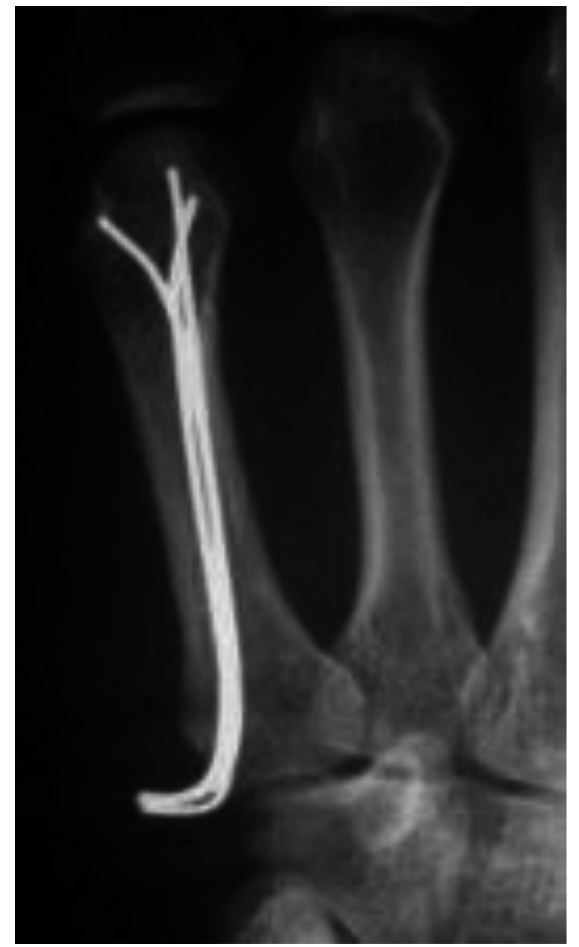

Figure 7. Bouquet Pinning Showing Reduction of the Metacarpal Neck Fracture by Three Antegrade Pins

placing the distal screws obliquely in different planes or by utilizing unicortical locking screws (Figure 8) (29).

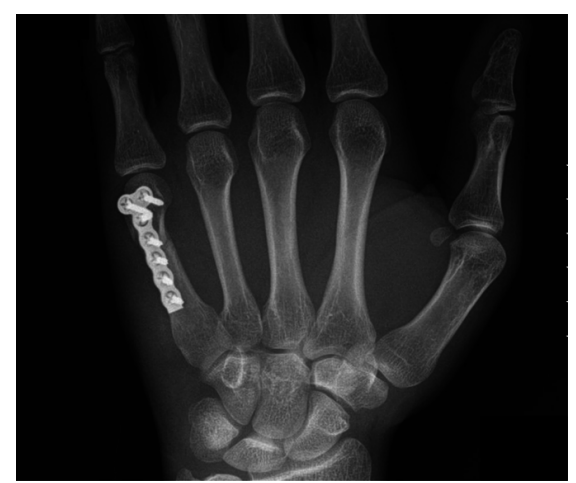

Figure 8. Plate and Screw Fixation of Metacarpal Neck Fracture

Benefits of the technique:

- Most biomechanically stable construct (36)

- Can be used to treat multiple concurrent metacarpal neck fractures (29)

- Can be used when significant comminution precludes closed reduction and percutaneous pinning (29)
Complications of the technique:

- Higher rates of stiffness/poor post-operative range of motion (58.7\% compared to contralateral side) $(33,37)$

- Metacarpal head avascular necrosis (33)

- Extensor tendon injury (17)

- Nonunion/malunion (37)

- Hardware irritation

\subsubsection{Intramedullary Fixation}

Distal fragment reduced by maximal flexion of the MCP joint with an axial load directed dorsally across the flexed PIP joint. Intramedullary fixation of the fracture can be accomplished with antegrade placement of pre-fabricated intramedullary nails (Figure 9) (29) or retrograde fixation with a headless compression screw placed through the metacarpal head (Figure 10).

Benefits of the techniques:

- Improved range of motion compared to k-wire crosspinning (38)

- Lower incidence of shortening compared to k-wire cross-pinning (38)

Complications/Drawbacks of the techniques:

- Nonunion/malunion (35)

- Limited rotational stability

\section{Conclusions}

Metacarpal neck fractures are common injuries of the hand. These injuries often result in missed time from work. In patients with the classic mechanism of injury of striking a solid surface, careful scrutiny for lacerations is critical as this may represent an open fracture. After diagnosis of a metacarpal neck fracture, surgery is indicated for open fractures, displaced intra-articular fractures, or in cases with unacceptable angulation. There is no consensus among hand surgeons as to the single most effective technique. As such, multiple fixation constructs exist as described with various risks and benefits that the clinician must weigh. While this review offers some guidance, the lack of an ideal surgical fixation technique necessitates further investigation of our current treatment strategies as well as the development of further novel techniques that may lead to improved outcomes. 
Figure 9. Intramedullary Fixation of Metacarpal Fractures With Commercially Available Nails Placed Antegrade
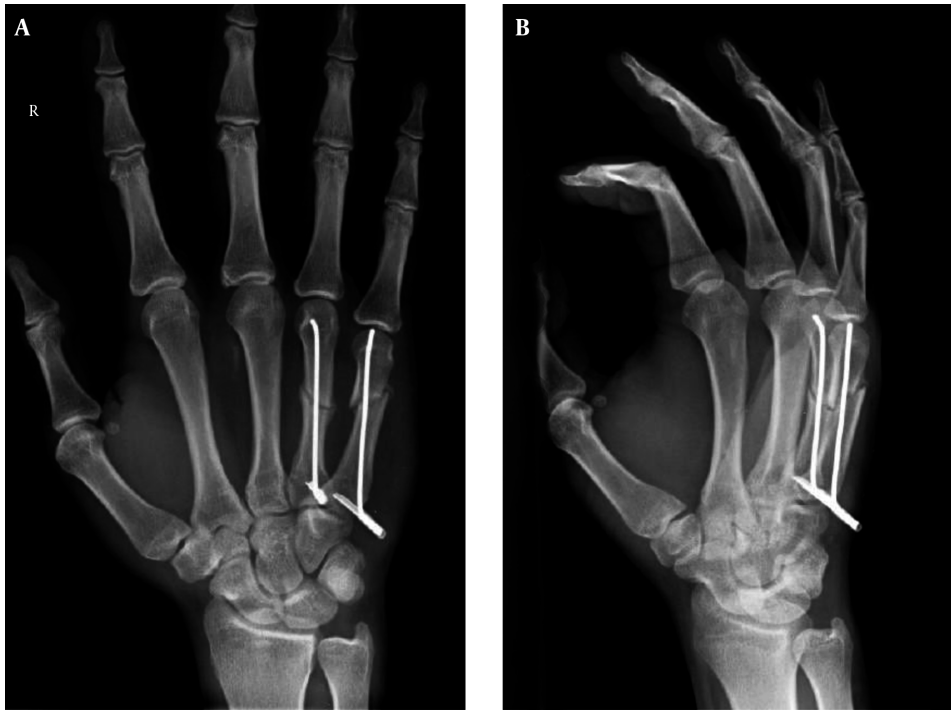

Later removal is required, (Courtesy Jorge Orbay, MD).

Figure 10. Fluoroscopic Assessment of Metacarpal Neck Fracture Treated by Reduction and Intramedullary Fixation With a Headless Compression Screw
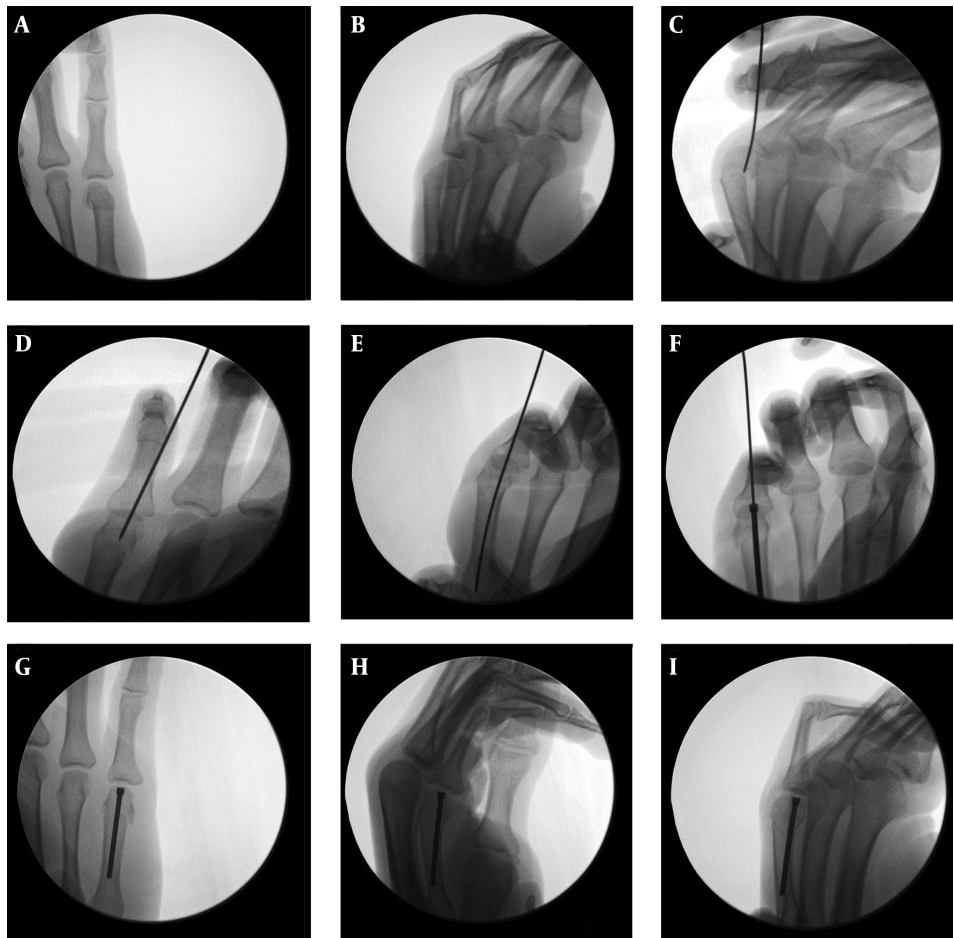

A and B, Intramedullary fixation with a headless compression screw is best indicated for neck and not shaft fractures, the technique requires exposure of the metacarpal head through a split in the extensor mechanism; C, D and E, the fracture is reduced and the guidewire is directed retrograde through the metacarpal head. Since the fracture typically flexes, the guidewire should be placed slightly volar in the metacarpal head as it is directed into the shaft, thereby aiding in reduction of the fracture by extending the fracture as the screw is inserted; F, the headless compression screw is then passed over the guidewire. The length of the screw should be measured so that it ends in the isthmus of the fracture and not the base in order to obtain good fixation while avoiding over-correction of the normal bow of the metacarpal; $\mathrm{G}$, $\mathrm{H}$ and $\mathrm{I}$, adequate reduction is held by one of these headless compression screws. 


\section{References}

1. Hile D, Hile L. The emergent evaluation and treatment of hand injuries. Emerg Med Clin North Am. 2015;33(2):397-408. doi: 10.1016/j.emc.2014.12.009. [PubMed: 25892728].

2. Meals C, Meals R. Hand fractures: a review of current treatment strategies. J Hand Surg Am. 2013;38(5):1021-31. doi: 10.1016/j.jhsa.2013.02.017. [PubMed: 23618458] quiz 1031.

3. Day C, Stern PJ. Fractures of the Metacarpals and Phalanges. 6th ed. Philadelphia: Elsevier Churchill Livingstone; 2011.

4. Ashkenaze DM, Ruby LK. Metacarpal fractures and dislocations. Orthop Clin North Am. 1992;23(1):19-33. [PubMed: 1729666].

5. Diao E. Metacarpal fixation. Hand Clin. 1997;13(4):557-71. [PubMed: 9403293].

6. Court-Brown CM, Caesar B. Epidemiology of adult fractures: A review. Injury. 2006;37(8):691-7. doi: 10.1016/j.injury.2006.04.130. [PubMed: 16814787].

7. Nakashian MN, Pointer L, Owens BD, Wolf JM. Incidence of metacarpal fractures in the US population. Hand (N Y). 2012;7(4):426-30. doi: 10.1007/s11552-012-9442-0. [PubMed: 24294164].

8. Statius Muller MG, Poolman RW, van Hoogstraten MJ, Steller EP. Immediate mobilization gives good results in boxer's fractures with volar angulation up to 70 degrees: a prospective randomized trial comparing immediate mobilization with cast immobilization. Arch Orthop Trauma Surg. 2003;123(10):534-7. doi: 10.1007/s00402-0030580-2. [PubMed: 14639483].

9. Harding IJ, Parry D, Barrington RL. The use of a moulded metacarpal brace versus neighbour strapping for fractures of the little finger metacarpal neck. J Hand Surg Br. 2001;26(3):261-3. doi: 10.1054/jhsb.2000.0509. [PubMed: 11386781].

10. van Aaken J, Kampfen S, Berli M, Fritschy D, Della Santa D, Fusetti C. Outcome of boxer's fractures treated by a soft wrap and buddy taping: a prospective study. Hand (N Y). 2007;2(4):212-7. doi: 10.1007/s11552007-9054-2. [PubMed: 18780055].

11. Jupiter JB, Goldfarb CA, Nagy L, Boyer MI. Posttraumatic reconstruction in the hand. J Bone Joint Surg Am. 2007;89(2):428-35. [PubMed: 17326313].

12. Henry M. Hand fractures and dislocations. Philadelphia: Lippincott Williams \& Wilkins; 2010.

13. Stanton JS, Dias JJ, Burke FD. Fractures of the tubular bones of the hand. J Hand Surg Eur Vol. 2007;32(6):626-36. doi: 10.1016/j.jhse.2007.06.017. [PubMed: 17993422].

14. Tosti R, Ilyas AM, Mellema JJ, Guitton TG, Ring D, Science of Variation G. Interobserver variability in the treatment of little finger metacarpal neck fractures. J Hand Surg Am. 2014;39(9):1722-7. doi: 10.1016/j.jhsa.2014.05.023. [PubMed: 25034789].

15. Beredjiklian PK. Small finger metacarpal neck fractures. $J$ Hand Surg Am. 2009;34(8):1524-6. doi: 10.1016/j.jhsa.2009.06.015. [PubMed: 19729252].

16. Strub B, Schindele S, Sonderegger J, Sproedt J, von Campe A, Gruenert JG. Intramedullary splinting or conservative treatment for displaced fractures of the little finger metacarpal neck? A prospective study. J Hand Surg Eur Vol. 2010;35(9):725-9. doi: 10.1177/1753193410377845. [PubMed: 20659966].

17. Page SM, Stern PJ. Complications and range of motion following plate fixation of metacarpal and phalangeal fractures. J Hand Surg Am. 1998;23(5):827-32. doi: 10.1016/S0363-5023(98)80157-3. [PubMed: 9763256]

18. Kollitz KM, Hammert WC, Vedder NB, Huang JI. Metacarpal fractures: treatment and complications. Hand (NY). 2014;9(1):16-23. doi: 10.1007/s11552-013-9562-1. [PubMed: 24570632].

19. Creighton Jr JJ, Steichen JB. Complications in phalangeal and metacarpal fracture management. Results of extensor tenolysis.
Hand Clin. 1994;10(1):111-6. [PubMed: 8188771].

20. Adkinson JM, Shafqat MS, Eid SM, Miles MG. Delayed diagnosis of hand injuries in polytrauma patients. Ann Plast Surg. 2012;69(4):4425. doi: 10.1097/SAP.0b013e31824b26e7. [PubMed: 22868310].

21. Perron AD, Miller MD, Brady WJ. Orthopedic pitfalls in the ED: fight bite. Am J Emerg Med. 2002;20(2):114-7. [PubMed: 11880877].

22. Shoji K, Cavanaugh Z, Rodner CM. Acute fight bite. J Hand Surg Am. 2013;38(8):1612-4. doi: 10.1016/j.jhsa.2013.03.002. [PubMed: 23660199].

23. Kamath JB, Harshvardhan , Naik DM, Bansal A. Current concepts in managing fractures of metacarpal and phalangess. Indian J Plast Surg. 2011;44(2):203-11. doi: 10.4103/0970-0358.85341. [PubMed: 22022030].

24. Thurston AJ. Pivot osteotomy for the correction of malunion of metacarpal neck fractures. J Hand Surg Br. 1992;17(5):580-2. [PubMed: 1479255]

25. Eyres KS, Allen TR. Skyline view of the metacarpal head in the assessment of human fight-bite injuries. J Hand Surg Br. 1993;18(1):43-4 [PubMed: 8436860].

26. Lane CS, Kennedy JF, Kuschner SH. The reverse oblique x-ray film: metacarpal fractures revealed. J Hand Surg Am. 1992;17(3):504-6. [PubMed: 1613232].

27. Leung YL, Beredjiklian PK, Monaghan BA, Bozentka DJ. Radiographic assessment of small finger metacarpal neck fractures.J Hand Surg Am. 2002;27(3):443-8. [PubMed: 12015718].

28. Lamraski G, Monsaert A, De Maeseneer M, Haentjens P. Reliability and validity of plain radiographs to assess angulation of small finger metacarpal neck fractures: human cadaveric study. J Orthop Res. 2006;24(1):37-45. doi: 10.1002/jor.20025. [PubMed: 16419967].

29. Leinberry C, Ukomadu U, Ilyas A. Metacarpal Fractures and Carpometacarpal Fracture-Dislocations. 1st ed. New Delhi, India: Jaypee Brothers Medical Publishers Ltd; 2013.

30. Ali A, Hamman J, Mass DP. The biomechanical effects of angulated boxer's fractures. J Hand Surg Am. 1999;24(4):835-44. [PubMed: 10447177].

31. Foucher G, Chemorin C, Sibilly A. [A new technic of osteosynthesis in fractures of the distal $3 \mathrm{~d}$ of the 5th metacarpus]. Nouv Presse Med. 1976;5(17):1139-40. [PubMed: 934828].

32. Winter M, Balaguer T, Bessiere C, Carles M, Lebreton E. Surgical treatment of the boxer's fracture: transverse pinning versus intramedullary pinning. J Hand Surg Eur Vol. 2007;32(6):709-13. doi 10.1016/j.jhse.2007.07.011. [PubMed: 17993437].

33. Facca S, Ramdhian R, Pelissier A, Diaconu M, Liverneaux $P$. Fifth metacarpal neck fracture fixation: Locking plate versus K-wire?. Orthop Traumatol Surg Res. 2010;96(5):506-12. doi: 10.1016/j.otsr.2010.02.009. [PubMed: 20580630].

34. Botte MJ, Davis JL, Rose BA, von Schroeder HP, Gellman H, Zinberg EM, et al. Complications of smooth pin fixation of fractures and dislocations in the hand and wrist. Clin Orthop Relat Res. 1992(276):194-201. [PubMed: 1537152]

35. Balaram AK, Bednar MS. Complications after the fractures of metacarpal and phalanges. Hand Clin. 2010;26(2):169-77. doi 10.1016/j.hcl.2010.01.005. [PubMed: 20494743].

36. Jones WW. Biomechanics of small bone fixation. Clin Orthop Relat Res. 1987(214):11-8. [PubMed: 3539435].

37. Fusetti C, Meyer H, Borisch N, Stern R, Santa DD, Papaloizos M. Complications of plate fixation in metacarpal fractures. J Trauma. 2002;52(3):535-9. [PubMed: 11901331].

38. Schadel-Hopfner M, Wild M, Windolf J, Linhart W. Antegrade in tramedullary splinting or percutaneous retrograde crossed pinning for displaced neck fractures of the fifth metacarpal?. Arch Orthop Trauma Surg. 2007;127(6):435-40. doi: 10.1007/s00402-006-0254-y. [PubMed: 17123093]. 